\title{
Nonextensive Effects in Tight-Binding Systems with Long-Range Hopping
}

\author{
Lisa Borland ${ }^{1}$ and J. G. Menchero ${ }^{2}$ \\ ${ }^{1}$ Centro Brasileiro de Pesquisas Fisicas (CBPF), \\ Rua Dr. Xavier Sigaud 150, 22090-180 Rio de Janeiro, Brazil. \\ ${ }^{2}$ Instituto de Fisica, Universidade Federal do Rio de Janeiro, \\ Cx. P. 68.528, 21945-970 Rio de Janeiro, Brazil
}

Received 07 December, 1998

\begin{abstract}
Consequences of long-range hopping in one-dimensional tight-binding models are studied. A hopping term proportional to $1 / r_{i j}^{\alpha}$ is used, where $r_{i j}$ denotes the distance between atoms $i$ and $j$ and $\alpha$ determines the range of the interactions within the system. Calculations of the diffusion of an electron along the lattice yield interesting effects of nonextensivity. In particular, we find that the mean square displacement scales anomalously as $D t^{\gamma}$ in the following way: For $0<\alpha<1$, we find $D \propto N N^{*}$, where $N$ is the number of atoms on the lattice and $N^{*}=\frac{N^{1-\alpha}-1}{1-\alpha}$ is related to the number of elements interacting at a given $\alpha$. In this regime the behaviour is subdiffusive $(.5 \leq \gamma<1)$ but approaches normal diffusion $(\gamma=1)$ for $\alpha=1$. There exists a transition region between $1<\alpha<2$, where the diffusion coefficient loses its system size dependency and becomes size independent for all $\alpha \geq 2$. In addition, we find $1<\gamma \leq 2$ (superdiffusion) for $\alpha>1$. Ballistic motion $(\gamma=2)$ is recovered for all $\alpha \geq 1.5$ and is maintained in the nearest neighbour limit. Specific heat and internal energy as a function of temperature and system size are also analyzed. They appear extensive on the macroscopic level for all values of $\alpha$.
\end{abstract}

\section{Introduction}

There is a current interest in studying systems with long-range microscopic interactions. Indeed, they have been found to exhibit a variety of interesting properties, ranging from nonextensive thermodynamic behaviour to anomalous diffusion and anomalous Lyapunov exponents ([1, 2, 3] and references therein). These results all imply that it may be neccessary to rethink the standard formulation of thermodynamics, which seems to break down when it comes to the description of this vast class of physical systems.

The generalized nonextensive thermostatistics recently proposed by Tsallis [4] has proven to be a successful candidate for treating a growing body of systems for which the standard thermodynamic formalism fails, and it appears to be that this is the framework neccessary to treat systems with long-range forces as well. Examples of situations where the generalized thermostatistics has been successfully applied - both theoretically and experimentally - are given by selfgravitating systems [5], two-dimensional turbulence in pure-electron plasma [6], the solar neutrino problem [7], nonlinear maps [8], anomalous diffusion of Lévy type [9] and correlated type [10] to name just a few.

In this paper we wish to study the effects of longrange hopping in a simple one-dimensional quantum mechanical tight-binding model of electrons on a lattice of atoms (see also [11]). This should be interesting since it is a quantum mechanical system and, although some studies for tight-binding electron models with long-range hopping do exist $[12,13]$, most of the systems studied with long-range interactions which we are aware of have been classical. Furthermore, models with long-range interactions have a close resemblence to other interesting physical problems as diverse as the Kondo effect [14] and neural systems modeling [15].

First, we shall give a brief background concerning certain scaling laws which have recently been proposed by Tsallis [16] for nonextensive systems. It is suggested that the integral

$$
N^{*}=d \int_{1}^{N^{1 / d}} d r r^{d-1} r^{-\alpha}=\frac{N^{1-\alpha / d}-1}{1-\alpha / d}
$$

governs the thermodynamic scalings of systems with power-law decaying interactions of type $1 / r^{\alpha}$. Here, $N$ 
is the system size, $d$ the dimension of the problem, $r$ a distance, and $\alpha$ a real number which determines the range of the interactions. In the limit $N \rightarrow \infty, N^{*}$ behaves as

$$
\begin{aligned}
\frac{1}{\alpha / d-1} & , \quad \alpha / d>1, \\
\ln N & , \quad \alpha / d=1, \\
\frac{N^{1-\alpha / d}}{1-\alpha / d} & , \quad 0 \leq \alpha / d<1 .
\end{aligned}
$$

It has been shown [16] that these conditions imply that the classical system is thermodynamically extensive for $\alpha / d>1$, wheras it becomes nonextensive for $0 \leq \alpha / d \leq 1$, and special scalings become necessary in order to have a mathematically and physically wellposed problem. For one-dimensional classical systems $(d=1)$, we expect the crossover from the extensive to the nonextensive regime to occur at the critical value $\alpha=1$. For quantum systems, it is as yet not quite clear where this crossover will occur. In addition, we point out that $N^{*}$ is essentially proportional to the number of elements interacting within the system at a particular range of the interactions, that is, at a particular value of $\alpha$. This can be seen most simply for $d=1$, as $N^{*}=\int_{1}^{N} d r r^{-\alpha}$ becomes just the integral of the probability $r^{-\alpha}$ of a particle interacting with another at distance $r$.

\section{The Model}

The system under study is a one-dimensional tightbinding model of electrons on a lattice with $N$ atomic sites, with a basis set of one s orbital per site. The tight-binding assumption implies that the electrons are localized on the lattice sites $i$, and the corresponding Hamiltonian has the form

$$
H=\sum_{i}^{N} \epsilon_{i} c_{i}^{+} c_{i}+\sum_{i, j \neq i}^{N} \frac{V}{r_{i j}^{\alpha}} c_{i}^{+} c_{j}+c c . .
$$

Here, the $c_{i}^{+}$and $c_{i}$ are creation and anhilation operators for electrons on site $i$ and the $\epsilon_{i}$ are the on-site energies, which are all set to zero. The power-law term $V / r_{i j}^{\alpha}$ describes the hopping of an electron from site $i$ to site $j$. With a lattice spacing equal to unity, the distance $r_{i j}$ will be measured in integer units. The parameter $\alpha$ determines the range of the interaction between different sites. It is clear that for $\alpha \rightarrow \infty$ we retrieve the conventional nearest neighbour (nn) model, whereas for $\alpha=0$ we obtain the mean-field limit where the electron can hop with equal probability to all sites.

\section{Static and Thermodynamic Properties}

\section{III.1 Energy Eigenvalues}

If we impose periodic boundary conditions then we obtain the following analytic expression for the energy eigenvalues:

$$
E_{k}=2 V \sum_{n=1} \frac{\cos (k n)}{n^{\alpha}},
$$

with $k=\pi-2 \pi(m-1) / N$ and $m=1, \cdots, N$. Here, $n$ corresponds to the integer distance between two sites $i$ and $j$. We assume that $N$ is an even number, and if we only consider the shortest distance between sites then the summation goes to $N / 2$ and one must subtract off half of the last term to avoid double counting.

We would like to point out that even though the results we obtain are almost exactly the same regardless of whether we use periodic boundary conditions or not, we choose to not use periodic boundary conditions in this paper. This is mainly because there may be some mathematical artifacts introduced when periodicity is imposed on systems with long-range interactions, which we wish to avoid. We will use Eq (6) only to illuminate some of our discussion later on. So instead of calculating the energy spectrum according to $\mathrm{Eq}(6)$, we find the energies by numerically diagonalizing the hopping matrix occurring in the Hamiltonian Eq (5).

The results are shown in Fig. 1a, where we see the energy eigenvalues for a few different values of the parameter $\alpha$, using a fixed number of atoms. The results obtained by the analytic formula in Eq (6) are very similar. Notice that for $\alpha \leq 1$, the lowest energy value $E_{\min }$ in the spectrum diverges, while all the others stay finite. We found that as the system size $N$ increases, the rate of this divergence scales exactly with the variable $N^{*}$ of $\mathrm{Eq}(1)$ leaving $E_{\min } / N^{*}$ constant for each value of $\alpha \leq 1$. This result is shown in Fig. 1b, and can be more easily understood with the aid of Eq (6). There we see that $E_{\min }$ is obtained for $k=0$, when all atoms are interacting constructively with all other atoms. This results in the energy term equal to

$$
E_{k}=2 V \sum_{n=1} \frac{1}{n^{\alpha}}
$$

where the sum, for large $N$, is none other than the discrete version of $N^{*}$ (see $\operatorname{Eq}(1)$ with $d=1$ ). For all 
the other eigenvalues, there are both constructive and destructive contributions to the total energy due to the different phase factors. Therefore, these do not diverge with increasing $N$ but stay finite even for $\alpha \leq 1$. It is worth remarking that this behaviour is very different from what would have been expected in most classical cases, where we would have expected a situation where all the energies diverge, and not just one.

\section{Energy Spectrum and Size Dependence}

(a)

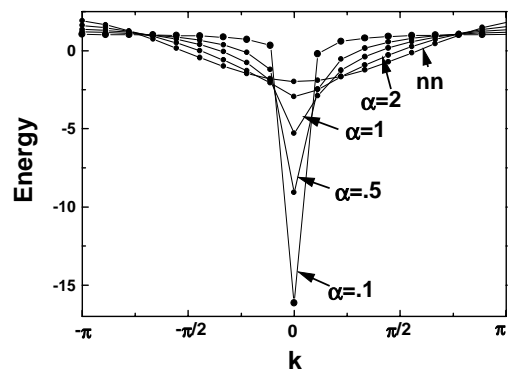

(b)

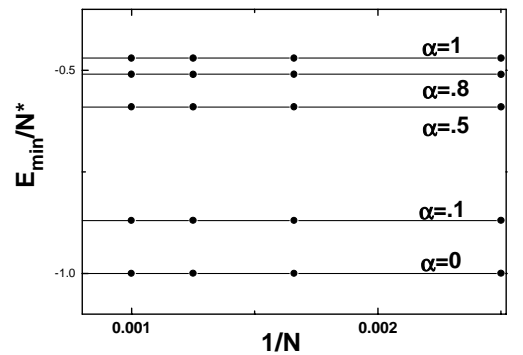

Figure 1. a) Energy levels for a system with 20 atoms (in units of $2 \mathrm{~V}$ ), calculated numerically without periodic boundary conditions, but plotted as a function of $k=$ $\pi(1-2(m-1) / N), \quad m=1 \cdots 20$. Here and in all Figures, $n n$ denotes the nearest neighbour limit of $\alpha \rightarrow \infty$. Note that the lowest energy level $E_{\text {min }}$ diverges for $\alpha \leq 1$, while all other energies remain finite. b) We found that the lowest energy level $E_{\min }$ diverges with system size such that $E_{\min } / N^{*}$ is constant for each given value of $\alpha \leq 1$.

\section{III.2 Specific Heat and Internal Energy}

Now we shall calculate some thermodynamic quantities such as the specific heat and internal energy. Let us assume that the system consists of a lattice of size $N$ atoms and $N / 2$ electrons, neglecting spin. At temperature $\tau=0$ the internal energy is given by

$$
U(0)=\sum_{i=1}^{N / 2} E_{i}
$$

where the $N / 2$ electrons occupy all states up to the Fermi level $E_{F}$. At a given temperature $\tau$ the internal energy becomes

$$
U(\tau)=\sum_{i=1}^{N} E_{i} f\left(E_{i}\right)
$$

which is simply the sum of energies of each state weighted by the probability $f\left(E_{i}\right)$ of that state being occupied. Here, $f\left(E_{i}\right)$ is given by the Fermi-Dirac distribution (with the Boltzmann constant $k_{B}$ set to 1 )

$$
f\left(E_{i}\right)=\frac{1}{\exp \left[\left(E_{i}-\mu(\tau)\right) / \tau\right]+1}
$$

where $\mu$ is the temperature dependent chemical potential which can be determined implicitly by

$$
N / 2=\sum_{i=1}^{N} f\left(E_{i}\right)=\sum_{i=1}^{N} \frac{1}{\exp \left[\left(E_{i}-\mu(\tau)\right) / \tau\right]+1} .
$$

We have plotted $\mu(\tau)$ in Fig. 2a, and in Fig. 2b we show $U(\tau) / N$ for different $N$. First of all, note that the internal energy $U$ is an extensive variable for all values of $\alpha$ at all temperatures, resulting in data collapse for all values of $N$. There is no dependence on $N^{*}$, even though we saw that the lowest energy $E_{\min }$ scales as $N^{*}$ with system size. This behaviour can be understood by rewriting the expression for the internal energy as $U(\tau)=\sum_{i=1}^{N} E_{i} f\left(E_{i}\right)=E-\sum_{i=1}^{N} E_{i}\left(1-f\left(E_{i}\right)\right)$, where $E$ is the sum over all energy levels $\sum_{i=1}^{N} E_{i}$ and is equal to a finite constant which we have chosen to be 0 . Now, $E_{\text {min }}$ is so low that it is always occupied with probability $f\left(E_{\text {min }}\right)=1$. However, in the second expression this implies that $E_{\text {min }}$ does not contribute to the sum at all. The internal energy is therefore determined only by the behaviour of all other energy levels, which remain finite and well behaved. As was seen in the energy spectrum (compare Fig. 1), those levels exhibit no noticeable change as we go from $\alpha>1$ to $\alpha \leq 1$, so it is not surprising that the same holds true for the internal energy. This result is very different from what we would have expected in the classical case, where it is predicted that the internal energy scales with the system size as $U /\left(N N^{*}\right)[16]$. 


\section{Chemical Potential, Specific Heat and Internal Energy}

(a)

3

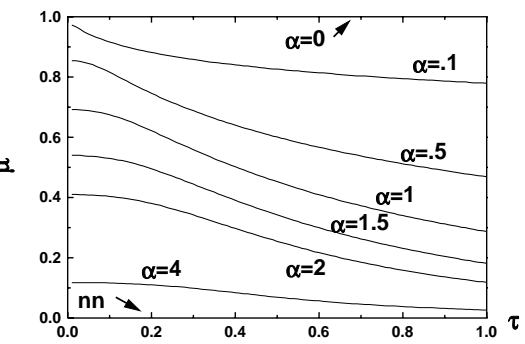

(b)

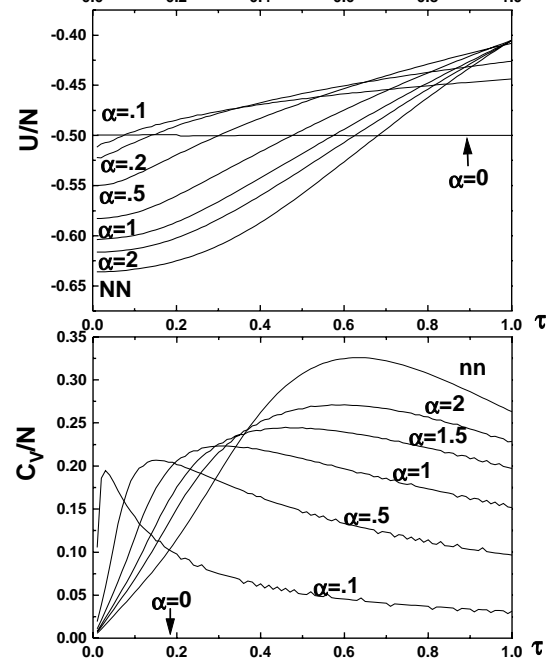

Figure 2: a) The chemical potential $\mu$ is plotted as a function of the temperature for different values of $\alpha$. The temperature $\tau$ is expressed in fractions of the characteristic interaction energy $V$. b) The internal energy $U / N$ is plotted as a function of temperature for different values of $\alpha$ and $N$. Again, all curves for different $N$ coincide. c) The specific heat $C_{V} / N$ as a function of temperature is shown for different values of $\alpha$ and different values of $N$. Note that there is data-collapse for all $N$, implying that $C_{V}$ is an extensive variable. (The fluctuations on the curves are numerical artifacts).

Next, we use the definition $C_{V}=\partial(U(\tau) / \partial \tau$ in conjunction with $\mathrm{Eq}(9)$ to obtain the specific heat of the system. $C_{V}(\tau) / N$ is plotted in Fig. 2c for different values of $\alpha$. The curves for different $N$ all coincide, implying that $C_{V}(\tau)$ is an extensive variable scaling with $N$ for all values of $\alpha$. The reason this is so despite the $N^{*}$ divergence of the lowest energy value is again because $E_{\min }$ is always occupied, so that any changes in the internal energy due to temperature will only involve the well behaved finite energy levels. In any case, it is interesting to note the marked difference in the temperature dependency of the specific heat for the different values of $\alpha$.

We calculated all of the above also using periodic boundary conditions, as well as varying the occupation level (i.e. number of electrons in the lattice). Neither of these variations affected our results in any significant way.

\section{Dynamic Properties}

\section{IV.1 Dispersion and Diffusion}

We now turn our attention from the static, thermodynamic properties to the dynamic, diffusive properties of the system. In contrast to the extensive behaviour observed for the thermodynamic properties, the diffusive properties show interesting nonextensive characteristics. There is a definite transition in behaviour as we go from the classically extensive $(\alpha>1)$ to the classically nonextensive $(\alpha \leq 1)$ regime.

At time $t=0$, a wavepacket may be expressed as

$$
\left|\psi(0)>=\sum_{i=1}^{N} a_{i}\right| f_{i}>,
$$

where the $a_{i}$ are the expansion coefficients with respect to the localized basis set $\left|f_{i}\right\rangle$, which are essentially just the sites of the atoms. We choose to start out with $\psi$ localized on a single atom at site $N / 2$ in the middle of the lattice, so that only $a_{N / 2} \neq 0=1$. Alternatively, we can represent the wavepacket $\psi$ in terms of the energy eigenbasis which we find numerically. Let us denote these eigenvectors by $\phi_{k}, k=1, \cdots N$. Then we get

$$
\left|\psi(0)>=\sum_{k=1}^{N} b_{k}\right| \phi_{k}>
$$

with the expansion coefficients $b_{k}=\sum_{i=1}^{N} a_{i}<\phi_{k} \mid$ $f_{i}>$. The time evolution of this wavepacket is given by

$$
\left|\psi(t)>=\sum_{k=1}^{N} b_{k}\right| \phi_{k}>e^{i E_{k} t / \hbar},
$$

which can be projected back onto the localized basis states to give the time-dependent amplitudes $a_{i}(t)=<$ $\psi(t) \mid f_{i}>$.

It is now straightforward to calculate the diffusion, which we define as the mean squared displacement of the position of the electron on the lattice. This is given by

$$
<x^{2}>(t)=\sum_{i} x_{i}^{2}\left|a_{i}(t)\right|^{2}
$$

where $\left|a_{i}(t)\right|^{2}$ gives the time-dependent probability of being on lattice site $i$, and $x_{i}$ is simply the position $i$ of the electron on the lattice, relative to the average position, i.e. $x_{i}=i-\langle i\rangle$ with $\langle i\rangle=\sum_{i} i\left|a_{i}(t)\right|^{2}$. 


\section{IV.2 Diffusion: Results and Analysis}

We expect the mean squared displacement of $\mathrm{Eq}$ (15) to follow the general diffusion equation

$$
<x^{2}>(t)=D t^{\gamma} .
$$

Here, $D$ is the diffusion coefficient and $\gamma$ the temporal diffusion exponent. If $\gamma=1$ the diffusion is said to be normal, if $\gamma<1$ the system is subdiffusive and $\gamma>1$ means that the system is superdiffusive. We shall now study the behaviour of both the diffusion coefficient $D$ and the exponent $\gamma$ for different values of $\alpha$ and system size $N$.

\section{Diffusion for Different $\alpha(\mathrm{N}=800)$}

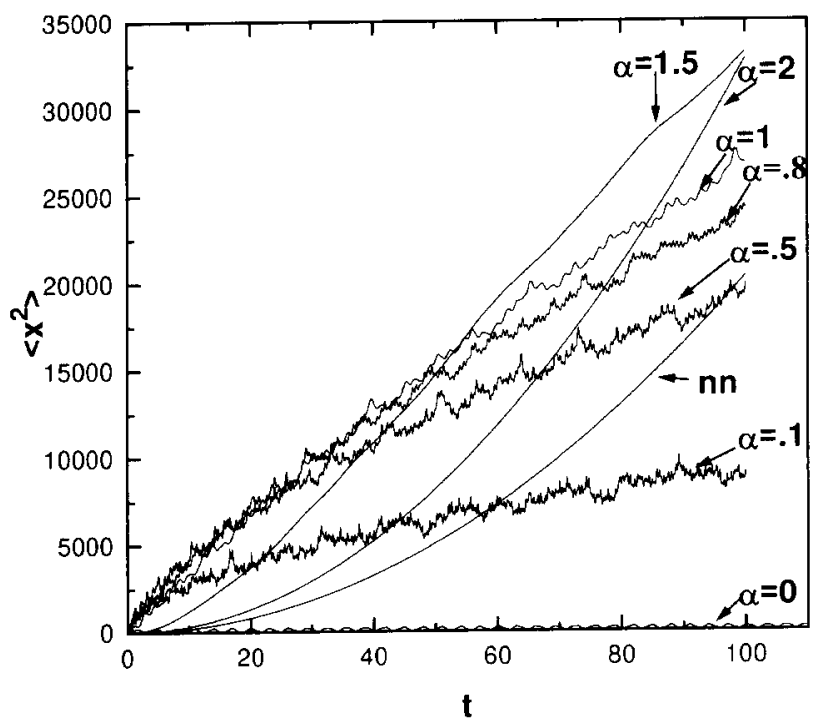

Figure 3: The mean squared displacement $\left\langle x^{2}\right\rangle$ (in units of lattice spacings) is plotted as a function of time ( $t$ given in units of $\hbar / V$ ) for fixed $N=800$ and different $\alpha$. There is a definite change in behaviour as $\alpha$ crosses the critical value of $\alpha=1$. In the regime $0 \leq \alpha \leq 1$ the diffusion goes from subdiffusive $(\gamma<1)$ to normal $(\gamma=1$, i.e. increasing linearly in time) yet with high-frequency oscillations. For $\alpha>1$, the oscillations disappear but the diffusion increases parabolically in time $(\gamma>1$, superdiffusion $)$. A more detailed analysis of $\gamma$ is presented in Figure 7 .

First, we leave the system size $N$ fixed and calculate the diffusion $\left\langle x^{2}\right\rangle(t)$ according to $\mathrm{Eq}(15)$ for different values of $\alpha$. These results are shown in Fig. 3, for timescales short enough to exclude finite size effects which will be discussed in Fig. 4. In the mean-field limit of $\alpha=0$ the wavepacket remains primarily localized on the initial site, with small temporal oscillations
[13]. As a consequence the mean squared deviation $\left\langle x^{2}\right\rangle(t)$ oscillates, but on the average the electron is trapped and does not diffuse at all. For small $\alpha$ close to 0 there is subdiffusive behaviour $(\gamma<1)$, but as $\alpha$ approaches 1 the diffusion appears to increase more or less linearly with time, i.e. with $\gamma \approx 1$. This corresponds to the case of normal diffusion. However, as $\alpha$ crosses the critical value of 1 , the diffusion is no longer linear in $t$. Instead it becomes parabolic, reaching a well-defined curve in the nearest-neighbour (nn) limit of $\alpha \rightarrow \infty$. We shall consider the analysis of $\gamma$ in more detail later on in Fig. 7. In addition, notice that all curves in the region $\alpha \leq 1$ exhibit high-frequency oscillating fluctuations, whereas these disappear for $\alpha>1$. We conjecture that these oscillations are of a similar nature to those seen in the mean-field limit.

It is clear from Fig. 3 that both $D$ and $\gamma$ depend on $\alpha$, but let us now see how the diffusion depends on $N$. To this end, we calculate the mean squared displacement $\left\langle x^{2}\right\rangle(t)$ for different values of $\alpha$ and $N$, a representative subset of which is shown in Fig. 4. Log-log plots of $\left\langle x^{2}\right\rangle(t)$ versus $t$ for $\alpha=.5$, which represents a typical case of $\alpha \leq 1$, is shown for different values of the system size $N$ in Fig. 4a. We show the calculations for very long times so as to exhibit the finite-size boundary effects, which can be seen in that that the curves all flatten out at large t. As $N$ increases the leveling off occurs at longer and longer times. To be most correct, our analysis of the diffusion should therefore be done at intermediate times where these boundary effects are negligible. Note also that the curves are distinctly seperated for different $N$. In Fig. 4b we plot the same as in Fig. 4a, except now for $\alpha=2$. Again we see the finite-size boundary effects in the leveling off of the diffusion curves, although now slight oscillations are exhibited in these tails. These oscillations do not exist for $\alpha<2$. However, the biggest difference to the $\alpha \leq 1$ case is that all the curves for different $N$ coincide, i.e., there is no divergence as $N$ increases. These results are typical for $\alpha \geq 2$, but not for the region $1<\alpha<2$ where we found yet another characteristic behaviour. Typical results are shown in Fig. $4 \mathrm{c}$ for $\alpha=1.5$. Again there is the finite-size flattening off, but the curves for different $N$ do not coincide, nor are they quite distinctly seperated for different $N$. 
(a)

(b)

(c)
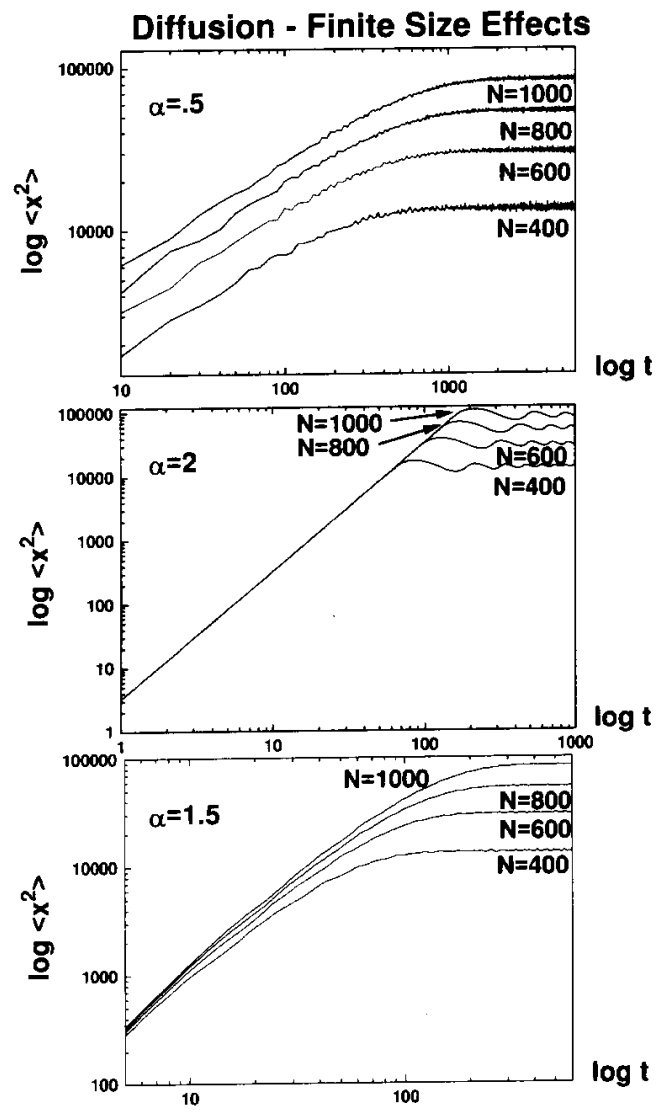

Figure 4: Log-log plots of the mean squared displacement (in units of lattice spacings) as a function of time (units of $\hbar / V$ ) are shown for different $\alpha$ and $N$. The leveling off at large times is a consequence of the finite-size boundary effects. Our studies should therefore be done at intermediate times where these effects are negligible. a) Typical results for $\alpha \leq 1$. The curves are distinctly seperated for different $N$. b) Typical results for $\alpha \geq 2$. The diffusion shows no size dependency. c) There exists a transition region $1<\alpha<2$ within which the curves lose their dependency on the system size $N$. Here at $\alpha=1.5$ it is clear that the curves neither coincide nor are quite distinctly seperated for different $N$.

The question which we now pose is whether any scaling laws or data-collapse can be obtained for the $N$-dependent diffusion curves. As shown in Fig. 5 this is achieved for all $\alpha \leq 1$ by plotting the quantity

$$
\frac{<x^{2}>}{N N^{*}}
$$

versus $t$ for different values of $N$ (here $N$ between $N=200$ and $N=1000$ ). These results imply that the diffusion coefficient $D$ scales as

$$
D=C_{\alpha} N N^{*} \quad \alpha \leq 1,
$$

where $C_{\alpha}$ denotes an $\alpha$-dependent constant. To our knowledge, such a size-dependent scaling law is re- ported for the first time here and in [11]. In [13] a sizedependency for the diffusion with $\alpha=1$ was discussed where scaling of the form $\left\langle x^{2}\right\rangle / N$ was suggested. For that particular value of $\alpha$ this is a reasonable approximation to our result Eq (18), which takes on the value $\left\langle x^{2}\right\rangle /(N \ln N)$ at $\alpha=1$. But it is definitely less accurate, and not at all valid for any other values of $\alpha$.

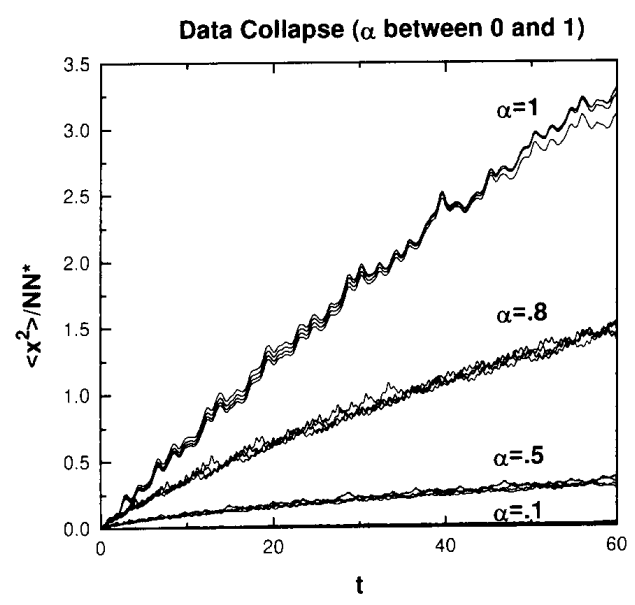

Figure 5: The renormalized quantity $\left\langle x^{2}\right\rangle /\left(N N^{*}\right)$ is plotted as a function of time (units of $\hbar / V$ ) for different values of $\alpha \leq 1$ and $N$ between 200 and 1000 , resulting in data-collapse for different $N$.

As was seen in Fig. 4b, there are no size-dependent effects in the region $\alpha \geq 2$. The diffusion coefficient can therefore be written as

$$
D=C_{\alpha} \quad \alpha \geq 2 .
$$

However, as we cross over the critical value of $\alpha=1$ into the regime $1<\alpha<2$, neither the scaling laws of $\mathrm{Eq}(18)$ or $\mathrm{Eq}$ (19) are valid. Instead it seems as though there is a continuous transition in this regime, where the curves for different values of $N$ become closer and closer together until they collapse onto one at the value $\alpha=2$. Data collapse was obtained empirically in this region, by renormalizing the diffusion curves by the function $N^{k(\alpha)}$, which is shown in Fig. 6. The function $\kappa(\alpha)$ was determined numerically from the data and is plotted in Fig. 6a. For $\alpha=1, N^{\kappa(\alpha)}=N^{1.16}$ which, for the sizes $N$ analysed (up to 1000), is close to the result $N N^{*}=N \ln N$ which we obtained by analysing the regime $0 \leq \alpha \leq 1$. For $\alpha \geq 1.5, \kappa(\alpha)$ becomes close to 0 , thus approaching the size independence which we 
observed for $\alpha \geq 2$. For large $N \rightarrow \infty$ the scaling represented by the function $\kappa(\alpha)$ will probably be an overestimation of the true size dependence. This can be seen for example by the fact that for $\alpha=1$ the scaling $N \ln N$ in the thermodynamic limit is better approximated by $N$ (i.e. $\kappa=1$ ) rather than $N^{1.16}$. In Fig. $6 \mathrm{~b}$ we show some renormalized data $\left\langle x^{2}\right\rangle / N^{\kappa}$. Note that although we do succeed in obtaining data collapse, finite size effects become apparent for larger times.

\section{Data Collapse for $1<\alpha<2$}

a)

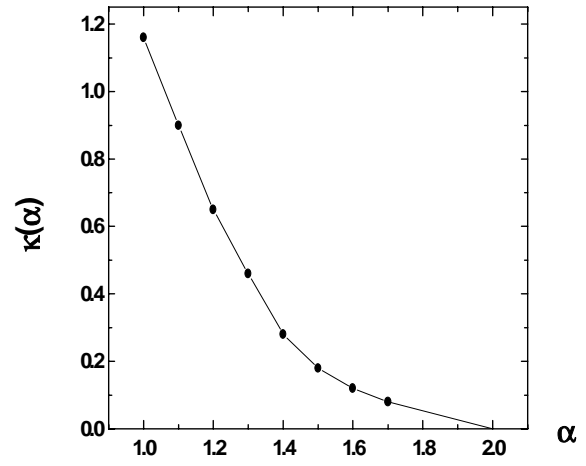

b)

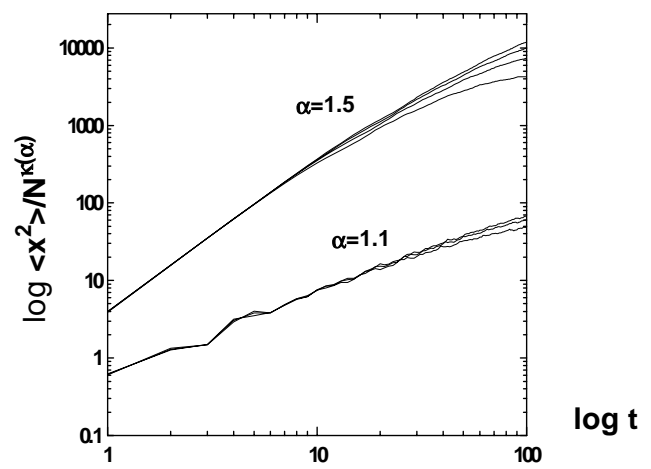

Figure 6: a) The scaling law $\left\langle x^{2}\right\rangle /\left(N N^{*}\right)$ is not valid in the regime $1<\alpha<2$. Instead we obtained data collapse by plotting $\left\langle x^{2}\right\rangle / N^{\kappa(\alpha)}$, using the function $\kappa$ shown here. $N$ ranges between 400 and 1000 in these curves. b) The normalized mean squared displacement $\left\langle x^{2}\right\rangle / N^{\kappa(\alpha)}$ is plotted in $\log -\log$ as a function of time (units of $\hbar / V$ ) for $\alpha=1.1$ and $\alpha=1.5$, demonstrating the data collapse obtained. Note that although we do succeed in collapsing the data onto a single curve, finite size effects become apparent for larger times.

So far we have mainly discussed the behaviour of the diffusion coefficent $D$. Let us now focus instead on the temporal dependency of $\left\langle x^{2}\right\rangle(t)$, which we briefly mentioned in conjunction with Figure 3. In Fig. 7 , we plot $\left\langle x^{2}\right\rangle(t)$ in $\log -\log$ so as to more accurately determine the diffusion exponent $\gamma$. In Fig. 7a we show the data $\left\langle x^{2}>(t)\right.$ for $\alpha \geq 1$. The slope of these curves is equal to $\gamma$, and we see that we have superdiffusive properties in this regime. The slopes of the curves vary from $\gamma=.9$ (which appears to go to normal diffusion of $\gamma=1$ as $N \rightarrow \infty$ ) for $\alpha=1$ to $\gamma=2$ (ballistic motion). Actually, all the curves for $\alpha>1$ start out with slope $\gamma \approx 2$ for small times, but for $\alpha \geq 1.5$ this time regime seems to dominate until finite size effects start entering, yielding $\gamma=2$ for $\alpha \geq 1$.5. In Fig. 7b we show the normalized data $\left\langle x^{2}\right\rangle /\left(N N^{*}\right)$ for different values of $\alpha \leq 1$. For $\alpha=0$ (not shown) the electron oscillates on the lattice but on the average is trapped and therefore does not diffuse at all. For $\alpha=.1$, we found that $\gamma \approx .5$, which is clearly subdiffusive behaviour. As $\alpha$ increases so does $\gamma$, reaching the value $\gamma=.9 \approx 1$ (normal diffusion) for $\alpha=1$.

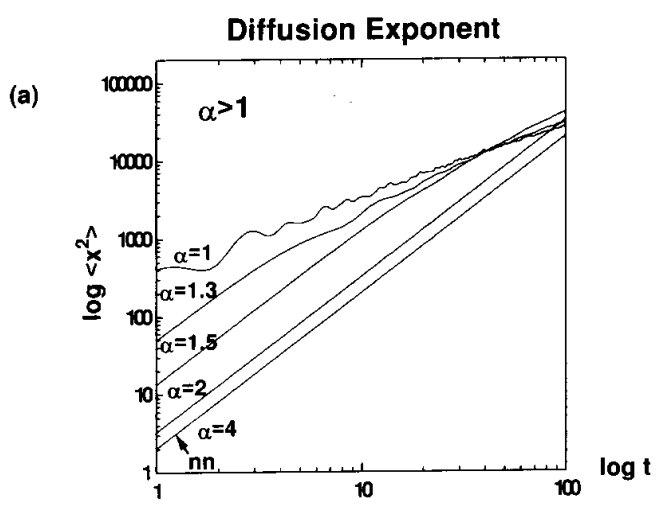

(b)

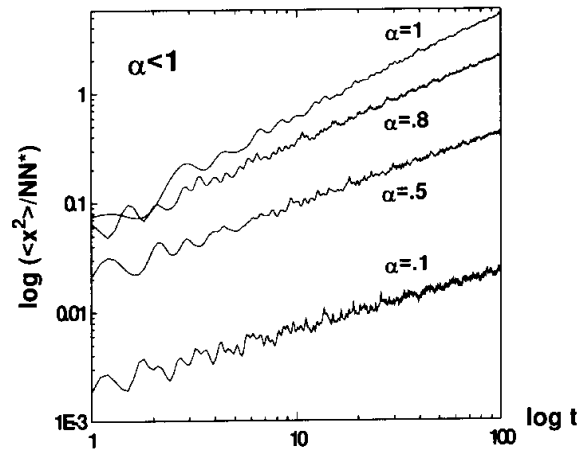

Figure 7: a) Log-log plots of $\left\langle x^{2}\right\rangle$ versus time (units of $\hbar / V$ ) are shown for different values of $\alpha \geq 1$. Curves are calculated using $N=800$. The slope of these curves is equal to the diffusion exponent $\gamma$, which varies from $\gamma=1$ to $\gamma=2$ in this regime. Note that all the curves for $\alpha>1$ seem to start out with slope $\gamma \approx 2$ for small times. Only for $\alpha \geq 1.5$ does this time regime seem to dominate until finite size effects become apparent. b) The renormalized data $\left\langle x^{2}\right\rangle /\left(N N^{*}\right)$ is plotted versus time for different $\alpha \leq 1$ using $N=800$. The slopes shown here vary from $\gamma \approx .5$ (subdiffusion) at $\alpha=.1$, to $\gamma=.9 \approx 1$ (normal diffusion) at $\alpha=1$. 
A summary of all of the above results is presented in Table I and in Fig. 8, where we show both $\gamma$ and the constant $C_{\alpha}$ as a function of $\alpha$. Fig. 8a shows $\gamma$ varying from .5 to 2 as a function of $\alpha$. In Fig. 8b the $\alpha$-dependent diffusion constant $C_{\alpha}$ is shown for the region $\alpha \leq 1$ where the diffusion coefficient behaves as $D=N N^{*} C_{\alpha}$. For $1<\alpha \leq 2$ the diffusion goes as $D=C_{\alpha} N^{\kappa(\alpha)}$ with $\kappa(\alpha)$ shown in Fig. 6 and $C_{\alpha}$ as shown in Fig. 8c. The constant $C_{\alpha}$ aquires a maximum value at $\alpha=1.5$. As of $\alpha \geq 2$ we obtain $D=C_{\alpha}$, and $C_{\alpha}$ (shown in Fig. $8 \mathrm{c}$ ) reaches the asymptotic value $C_{\alpha}=2$ for all $\alpha \geq 4$.

\section{Summary and Conclusions}

In this paper we have studied the consequences of long-range interactions in a quantum mechanical tightbinding model of electrons on a one dimensional lattice. A hopping term proportional to $1 / r_{i j}^{\alpha}$ was used, where $r_{i j}$ denotes the distance between atoms $i$ and $j$ and $\alpha$ determines the range of the interactions within the system. Both thermodynamic and diffusive properties were studied, as a function of the range of the interactions and of the system size. We found that only the minimum energy level $E_{\text {min }}$ diverges as $\alpha$ crosses over from the classically extensive regime $(\alpha>1)$ to the classically nonextensive regime $(\alpha<1)$. Furthermore we were able to determine that this divergence is such that $E_{\min } / N^{*}$ remains finite and constant for each $\alpha<1$, with $N^{*}$ given by Eq (1). This makes sense because, for $\alpha \leq 1, N^{*}$ is proportional to the number of particles interacting with each other, and the energy eigenvalue which diverges is one which consists of constructive contributions from all interacting atoms. All other eigenvalues remain finite because they contain both constructive and destructive contributions due to the different phase factors associated with the different atoms.

Macroscopic thermodynamic quantities such as the internal energy and specific heat were calculated as a function of temperature and system size $N$. Somewhat surprisingly, they both appeared to be extensive for all values of $\alpha$, showing no dependency on $N^{*}$ despite the divergence of $E_{\min }$ for $\alpha<1$. This can be understood as a consequence of the fact that the diverging state is essentially always occupied. This implies, for example, that the specific heat can only depend on the other energy levels, which are all finite and well-behaved for all values of $\alpha$. Our results are very different from the classical case where, for instance, the internal energy is predicted to scale as $N N^{*}$. This difference is probably due to the fact that in most classical settings we would expect all energies to scale as $E /\left(N N^{*}\right)$ [16] and not just one as $E / N^{*}$.

We then studied the diffusion of an electron along the lattice, and found large differences in behaviour depending on the value of $\alpha$. These are summarized in Table I and in Fig. 8. We report here, for the first time to our knowledge, that the diffusion coefficient diverges as $D=C_{\alpha} N N^{*}$ in the regime $\alpha<1$, where $C_{\alpha}$ is an $\alpha$-dependent constant. Furthermore, the diffusion in this regime ranges from subdiffusive $(\gamma<1)$ to normal $(\gamma=.9 \approx 1)$, and exhibits high frequency oscillations. The behaviour changes at the critical value of $\alpha=1$, after which there is superdiffusion $(1<\gamma \leq 2)$. The value of $\gamma=2$, which corresponds to ballistic motion, is reached already for $\alpha=1.5$. In addition, the scaling of the diffusion coefficent undergoes a transition from size-dependent to size-independent as $\alpha$ goes from 1 to 2. For $\alpha \geq 2, D$ is completely independent of the system size.

Table I $-\left\langle x^{2}\right\rangle(t)=D t^{\gamma} \quad D$ and $\gamma$ as a function of $\alpha$

\begin{tabular}{|l|l|ll|}
$\alpha$ & $D$ & $\gamma$ & \\
\hline$\alpha=0(\mathrm{mf})$ & $D=0$ & & no diffusion \\
$0<\alpha<1$ & $D=C_{\alpha} N N^{*}$ & $.5 \geq \gamma<1$ & subdiffusion \\
$\alpha=1$ & $D=C_{\alpha} N \ln N$ & $\gamma \approx 1$ & normal diffusion \\
$1<\alpha<2$ & $D=C_{\alpha} N^{k(\alpha)}$ & $1 \leq \gamma \leq 2$ & superdiffusion \\
$\alpha \geq 2$ & $D=C_{\alpha}$ & $\gamma=2$ & ballistic motion \\
$\alpha \rightarrow \infty(\mathrm{nn})$ & $D=C_{\alpha}$ & $\gamma=2$ & ballistic motion
\end{tabular}

(Note: $\kappa(\alpha)$ as in Fig. 6a.) 
a)

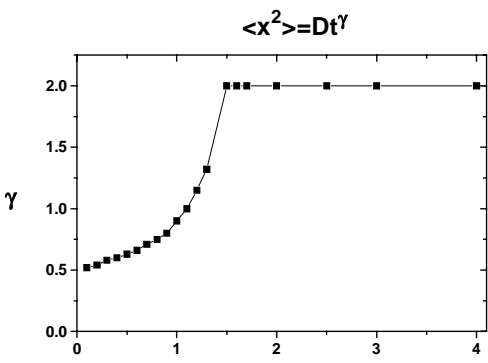

b)

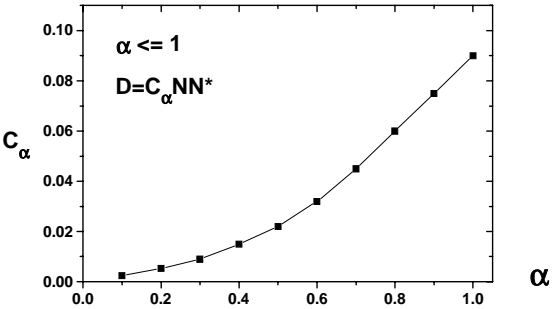

c)

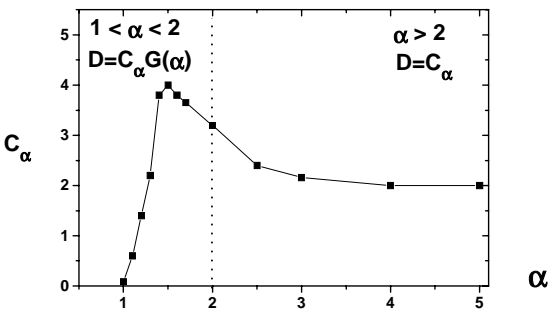

Figure 8. a) The diffusion exponent $\gamma$ is shown here as a function of the parameter $\alpha$, which determines the range of the interactions. For $0<\alpha \leq 1$ there is subdiffusion with $\gamma$ ranging from $\gamma=.5$ for small $\alpha$ almost up to normal diffusion with $\gamma=.9$ for $\alpha=1$. The regime $\alpha>1$ exhibits superdiffusive behaviour $\gamma>1$, approaching ballistic motion $\gamma=2$ at $\alpha \approx 1.5$ and beyond. b) For $\alpha \leq 1$ the diffusion coefficient $D$ is size-dependent such that $D=N N^{*} C_{\alpha}$. Here, we show how $C_{\alpha}$ varies. c) For $1<\alpha \leq 2$ the diffusion goes as $D=C_{\alpha} N^{\kappa(\alpha)}$ with $\kappa(\alpha)$ shown in Fig. 6 and $C_{\alpha}$ as shown here. A maximum is obtained at $\alpha=1.5$. As of $\alpha \geq 2$ we obtain $D=C_{\alpha} . C_{\alpha}$ reaches the asymptotic value 2 at about $\alpha=4$.

These results deserve a little more discussion. In particular it is interesting to note the existence of three regimes of behaviour. This is analogous to results found for one-dimensional classical spin-systems [2], where there is a crossover from nonextensive to extensive behaviour at $\alpha=1$, yet there are two behavioural regimes within the extensive $\alpha>1$ parameter region. One of these is for $1<\alpha<2$ and the other is for $\alpha \geq 2$, which coincides with our results found here. This existence of two distinct extensive regimes is a phenomenon only seen in one-dimensional systems. However, in the present quantum case, it may be that the real crossover from nonextensive to extensive behavior occurs at a value different from that in the classical case, because the behavior in the $1<\alpha<2$ regime shows some nonextensive scaling effects. Our work indicates that the quantum crossover may therefore be for $\alpha$ as large as $\alpha=d+1=2$.

Another interesting point which is as yet unclear is why the diffusion coefficient diverges with $N N^{*}$ for $\alpha \leq 1$. This may be related to the fact that we are considering the diffusion of a single electron on the lattice, so that the lowest diverging energy level is likely to have a large influence. The transition from subdiffusive to superdiffusive behaviour as we pass from the nonextensive to the classically extensive regimes is also not understood. However, both of these effects clearly must be investigated in greater detail.

In summary, we may say that the range of the hopping has considerable consequences for one-dimensional tight-binding systems. Though the thermodynamic properties of specific heat and internal energy appear largely unaffected with respect to their extensive behaviour, the diffusive properties change drastically. It would surely be interesting to explore the behaviour of even more quantities for this simple system, and also to study the effects of long-range interactions in other quantum mechanical models.

\section{Acknowledgements}

The authors wish to thank Constantino Tsallis for initiating and inspiring this work through many helpful discussions. JGM acknowledges financial support from the Brazilian funding agency CNPq. LB acknowledges that this material is based upon work supported by the National Science Foundation under Grant No. INT-9703649. Infrastructural benefits from a PRONEX grant (Brazilian agency) are also acknowledged.

\section{References}

[1] P. Jund, S.G. Kim and C. Tsallis, Phys. Rev. B 52, 50 (1995).

[2] S.A. Cannas and F.A. Tamarit, Phys. Rev. B 54 , R12661 (1996).

[3] C. Anteneodo and C. Tsallis, Phys. Rev. Lett. 80, 5313 (1998).

[4] C. Tsallis, J. Stat. Phys. 52, 479 (1988); E.M.F. Curado and C. Tsallis, J. Phys. A 24, L69 (1991).

[5] A.R. Plastino and A. Plastino, Phys. Lett. A 174, 384 (1993).

[6] B.M. Boghosian, Phys. Rev. E 53, 4754 (1996).

[7] G. Kaniadakis, A. Lavagno and P. Quarati, Phys. Lett. B 369, 308 (1996). 
[8] U.M.S. Costa, M.L. Lyra, A.R. Plastino and C. Tsallis, Phys. Rev. E 26, 245 (1997); M.L. Lyra and C. Tsallis, Phys. Rev. Lett. 80, 53 (1998).

[9] P.A. Alemany and D.H. Zanette, Phys. Rev. E 49, R956 (1994); D.H. Zanette and P.A. Alemany, Phys. Rev. Lett. 75, 366 (1995); C. Tsallis, S.V.F. Levy, A.M.C. Souza and R. Maynard, Phys. Rev. Lett. 75, 3589 (1995) [Erratum: Phys. Rev. Lett. 27, 5442 (1996)].

[10] A.R.Plastino and A.Plastino, Physica A 222, 347 (1995); C. Tsallis and D.J. Bukman, Phys. Rev. E 54, R2197 (1996); L. Borland, Phys. Rev. E 57, 6634 (1998).

[11] L. Borland, J.G. Menchero and C. Tsallis Anomalous diffusion and nonextensive scaling in a one- dimensional quantum model with long-range interactions, unpublished (1998).

[12] J.C. Cressoni and M.L. Lyra, The nature of electronic states in a disordered chain with long-ranged hopping amplitudes, Physica A, in press.

[13] H.N. Nazareno and P.E. de Brito Long-range interactions and nonextensivity in one-dimensional systems, Preprint (1998).

[14] P.W. Anderson and G. Yuval, Phys. Rev. B 1, 1522 (1970);

[15] D.J. Amit, Modeling Brain Functions, (Cambridge University Press, 1989).

[16] C. Tsallis, Fractals 3, 541 (1995). 